STUPP-92-130

August, 1992

\title{
Semiclassical Quantization of Two-Dimensional Dilaton Gravity
}

\author{
YOSHIAKI TANII \\ Physics Department, Saitama University \\ Urawa, Saitama 338, Japan
}

\begin{abstract}
Quantization of the dilaton gravity in two dimensions is discussed by a semiclassical approximation. We compute the fixed-area partition function to one-loop order and obtain the string susceptibility on Riemann surfaces of arbitrary genus. Our result is consistent with the approach using techniques of conformal field theories.
\end{abstract}


A two-dimensional metric-dilaton system coupled to matter fields (dilaton gravity) was proposed in refs. [1, 2] as a simple model to discuss the quantum theory of black holes. Although this model is exactly solvable at the classical level, the quantum theory is not yet fully understood.

Recently, quantization of this model was discussed in refs. [3, 4] using techniques of conformal field theories. (Quantization was also discussed using other methods in ref. [5].) The authors in refs. [3, 4] made an ansatz about the functional measures of path integrals following a procedure applied to ordinary two-dimensional gravity in ref. [6]. In the case of ordinary gravity the ansatz was justified by comparing its results with those of the matrix models [7]. The ansatz was also checked by other approaches such as semiclassical analyses 8, 9], the light-cone gauge quantization [10] and direct calculations of the functional measures [11]. Since matrix models for the dilaton gravity are not known at present, it is important to study other approaches and compare their results with those of refs. [3, 4].

The purpose of the present paper is to study the dilaton gravity by a semiclassical approximation, which becomes exact for a matter central charge $c \rightarrow-\infty$. We find a saddle point of the path integral with a fixed 'area' and quantize fluctuations of fields around it to one-loop order. In particular, we compute the string susceptibility on Riemann surfaces of arbitrary genus. Our result is consistent with that of refs. [3, 4. We also study a case in which the functional measures are modified as in ref. [3.

We consider a conformal field theory with a central charge $c$ coupled to a metricdilaton system on a compact closed surface. The metric is chosen to have the Euclidean signature. The classical action is

$$
S=\frac{1}{2 \pi} \int d^{2} \xi \sqrt{g} \mathrm{e}^{-2 \phi}\left(R_{g}+2 \mu+4 g^{\alpha \beta} \partial_{\alpha} \phi \partial_{\beta} \phi\right)+S_{\mathrm{M}}\left[g_{\alpha \beta}, f\right]
$$

where $\phi$ is the dilaton field, $R_{g}$ is the scalar curvature of the metric $g_{\alpha \beta}$ and $S_{\mathrm{M}}$ is the action for the matter field $f$. The parameter $\mu$ is a generalized cosmological 
constant. We have chosen the sign of the metric-dilaton terms in eq. (11) opposite to that of ref. [四]. This choice of the sign is convenient for a semiclassical analysis in the limit $c \rightarrow-\infty$. The partition function on the genus $h$ Riemann surface is given by a path integral

$$
Z_{\chi}(\mu)=\int \frac{\mathcal{D}_{g} g_{\alpha \beta} \mathcal{D}_{g} \phi \mathcal{D}_{g} f}{V_{\text {gauge }}} \mathrm{e}^{-S}
$$

where $\chi=2-2 h$ is the Euler number of the surface and $V_{\text {gauge }}$ is the volume of the group of diffeomorphisms. The functional measures are defined in a diffeomorphism invariant way using the metric $g_{\alpha \beta}$. In particular, the measures of the metric and the dilaton are defined by the norms 12

$$
\begin{aligned}
\left\|\delta g_{\alpha \beta}\right\|_{g}^{2} & =\int d^{2} \xi \sqrt{g}\left(g^{\alpha \gamma} g^{\beta \delta}+u g^{\alpha \beta} g^{\gamma \delta}\right) \delta g_{\alpha \beta} \delta g_{\gamma \delta} \quad\left(u>-\frac{1}{2}\right) \\
\|\delta \phi\|_{g}^{2} & =\int d^{2} \xi \sqrt{g}(\delta \phi)^{2}
\end{aligned}
$$

In ref. [3] a different choice of the measures was used. We will discuss such modifications of the measures at the end of the paper. For a semiclassical analysis it is more convenient to consider the partition function with a fixed 'area' $A$

$$
\tilde{Z}_{\chi}(A)=\int \frac{\mathcal{D}_{g} g_{\alpha \beta} \mathcal{D}_{g} \phi \mathcal{D}_{g} f}{V_{\text {gauge }}} \delta\left(\int d^{2} \xi \sqrt{g} e^{-2 \phi}-A\right) \mathrm{e}^{-S}
$$

The partition function (2) is obtained from eq. (4) by integrating over $A$.

To fix the diffeomorphism invariance we choose the conformal gauge 12

$$
g_{\alpha \beta}(\xi)=\mathrm{e}^{2 \rho(\xi)} \hat{g}_{\alpha \beta}(\xi ; \tau)
$$

where $\hat{g}_{\alpha \beta}$ is a reference metric which depends on the moduli $\tau$ of the Riemann surface. After the gauge fixing and an integration of the matter field we obtain

$$
\tilde{Z}_{\chi}(A)=\int \frac{[d \tau]}{V_{\mathrm{CKV}}} \int \mathcal{D}_{g} \rho \mathcal{D}_{g} \phi \delta\left(\int d^{2} \xi \sqrt{\hat{g}} e^{2(\rho-\phi)}-A\right) \mathrm{e}^{-S_{\mathrm{eff}}}
$$


where $V_{\mathrm{CKV}}$ is the volume of the group generated by the conformal Killing vectors, which exist for genera $h=0,1$. The effective action is given by

$$
S_{\text {eff }}=\frac{1}{2 \pi} \int d^{2} \xi \sqrt{\hat{g}}\left[\mathrm{e}^{-2 \phi}\left(R_{\hat{g}}-4 \hat{g}^{\alpha \beta} \partial_{\alpha} \phi \partial_{\alpha} \rho+4 \hat{g}^{\alpha \beta} \partial_{\alpha} \phi \partial_{\beta} \phi\right)\right]+S_{\mathrm{L}}+\frac{\mu}{\pi} A,
$$

where we have used the fixed area condition. The Liouville action

$$
S_{\mathrm{L}}\left[\hat{g}_{\alpha \beta}, \rho\right]=\frac{\gamma}{2 \pi} \int d^{2} \xi \sqrt{\hat{g}}\left(\hat{g}^{\alpha \beta} \partial_{\alpha} \rho \partial_{\beta} \rho+R_{\hat{g}} \rho+2 \mu^{\prime} \mathrm{e}^{2 \rho}\right), \quad \gamma=\frac{26-c}{12}
$$

is a result of the Weyl anomaly of the matter and the Faddeev-Popov ghost fields [12]. The parameter $\mu^{\prime}$ is regularization dependent and we choose it to be zero for simplicity.

The functional measures of $\rho$ and $\phi$ in eq. (6) are defined by the norms induced from eq. (3)

$$
\begin{aligned}
\|\delta \rho\|_{g}^{2} & =\int d^{2} \xi \sqrt{\hat{g}} \mathrm{e}^{2 \rho}(\delta \rho)^{2}, \\
\|\delta \phi\|_{g}^{2} & =\int d^{2} \xi \sqrt{\hat{g}} \mathrm{e}^{2 \rho}(\delta \phi)^{2} .
\end{aligned}
$$

Due to the factor $\mathrm{e}^{2 \rho}$ in these norms it is not obvious how to evaluate the functional integral. In refs. [3, [1] a relation of these measures to those without the factor $\mathrm{e}^{2 \rho}$ was given as an ansatz following the procedure in ref. [6]. We will check this ansatz by evaluating the functional integral (6) in a semiclassical approximation, which becomes exact for $c \rightarrow-\infty$.

As a first step of the semiclassical quantization let us find a saddle point of the functional integral (6). We have to find a minimum of the exponent $-S_{\text {eff }}$ under the condition of fixed area. Introducing the Lagrange multiplier $\lambda \in \mathbf{R}$ it can be found by the variational principle of $S_{\text {eff }}+\frac{\lambda}{\pi}\left(\int d^{2} \xi \sqrt{\hat{g}} e^{2(\rho-\phi)}-A\right)$. The Euler-Lagrange equations are

$$
2 \Delta_{\hat{g}} \rho-R_{\hat{g}}-2 \lambda \mathrm{e}^{2 \rho}-4\left(\Delta_{\hat{g}} \phi-\hat{g}^{\alpha \beta} \partial_{\alpha} \phi \partial_{\beta} \phi\right)=0
$$




$$
\begin{aligned}
2 \Delta_{\hat{g}} \rho-R_{\hat{g}}-\frac{4 \lambda}{\gamma} \mathrm{e}^{2(\rho-\phi)}-\frac{4}{\gamma}\left(\Delta_{\hat{g}} \phi-\hat{g}^{\alpha \beta} \partial_{\alpha} \phi \partial_{\beta} \phi\right) e^{-2 \phi} & =0, \\
\int d^{2} \xi \sqrt{\hat{g}} e^{2(\rho-\phi)}-A & =0 .
\end{aligned}
$$

If we choose the dilaton field to be a constant

$$
\phi_{\mathrm{cl}}=-\frac{1}{2} \ln \frac{\gamma}{2}
$$

then the first two equations of eq. (10) are reduced to a single equation

$$
R_{\bar{g}}=-2 \lambda, \quad \bar{g}_{\alpha \beta} \equiv \mathrm{e}^{2 \rho_{\mathrm{cl}}} \hat{g}_{\alpha \beta},
$$

which expresses the fact that the metric $\bar{g}_{\alpha \beta}$ has a constant curvature. The multiplier $\lambda$ can be fixed by integrating eq. (12) over the surface and using the third equation of eq. (10)

$$
\lambda=-\frac{\pi \gamma \chi}{A}
$$

It is a mathematical theorem that there exists a unique metric with a constant curvature on any Riemann surface. Thus eq. (12) with eq. (13) determines $\rho_{\mathrm{cl}}$. It is easy to see that the $A$-dependence of $\rho_{\mathrm{cl}}$ is

$$
\rho_{\mathrm{cl}}=\rho_{\mathrm{cl}}^{A=1}+\frac{1}{2} \ln A
$$

If we considered the partition function (2) instead of the area-fixed one (4), the saddle point condition would be the first two equations of eq. (10) with $\lambda$ replaced by the cosmological constant $\mu$. Then, as one can easily see by integrating the equation corresponding to eq. (12), a solution of the form (11), (12) is possible only for either $\chi>0$ or $\chi<0$ depending on the sign of $\mu$. This is the reason why we have considered the area-fixed partition function (4).

Next we define quantum fluctuations around the above configuration

$$
\tilde{\phi}=\phi-\phi_{\mathrm{cl}}, \quad \tilde{\rho}=\rho-\rho_{\mathrm{cl}}
$$


and expand the action $S_{\text {eff }}$ in these fluctuations. The action up to quadratic terms is

$$
\begin{aligned}
S_{\text {eff }}= & \frac{\mu}{\pi} A+\gamma \chi+S_{\mathrm{L}}\left[\hat{g}_{\alpha \beta}, \rho_{\mathrm{cl}}^{A=1}\right]+\gamma \chi \ln A+\frac{\gamma}{2 \pi} \int d^{2} \xi \sqrt{\bar{g}}\left[\bar{g}^{\alpha \beta} \partial_{\alpha} \tilde{\phi} \partial_{\beta} \tilde{\phi}\right. \\
& \left.+\frac{2 \pi \gamma \chi}{A} \tilde{\phi}^{2}+\bar{g}^{\alpha \beta} \partial_{\alpha}(\tilde{\rho}-\tilde{\phi}) \partial_{\beta}(\tilde{\rho}-\tilde{\phi})-\frac{2 \pi \gamma \chi}{A}(\tilde{\rho}-\tilde{\phi})^{2}\right] .
\end{aligned}
$$

To obtain eq. (16) we have used the fixed area condition

$$
\begin{aligned}
0 & =\int d^{2} \xi \sqrt{\hat{g}} \mathrm{e}^{2(\rho-\phi)}-A \\
& =\gamma \int d^{2} \xi \sqrt{\bar{g}}\left[(\tilde{\rho}-\tilde{\phi})+(\tilde{\rho}-\tilde{\phi})^{2}+\cdots\right]
\end{aligned}
$$

to eliminate linear terms in the expansion.

We now substitute eq. (16) into eq. (6) and evaluate the integrals. We decompose the fields into zero (constant) modes $\rho_{0}, \phi_{0}$ and nonzero modes $\rho^{\prime}, \phi^{\prime}\left(\tilde{\rho}=\rho_{0}+\right.$ $\left.\rho^{\prime}, \tilde{\phi}=\phi_{0}+\phi^{\prime}\right)$ with respect to the Laplacian $\Delta_{\bar{g}}$. To the one-loop order the norm of $\rho(9)$ can be approximated as 8,9

$$
\begin{aligned}
\|\delta \rho\|_{g}^{2} & =\int d^{2} \xi \sqrt{\bar{g}} \mathrm{e}^{2 \tilde{\rho}}(\delta \tilde{\rho})^{2} \\
& \cong \int d^{2} \xi \sqrt{\bar{g}}(\delta \tilde{\rho})^{2} \\
& =\left(\delta \rho_{0}\right)^{2} \int d^{2} \xi \sqrt{\bar{g}}+\int d^{2} \xi \sqrt{\bar{g}}\left(\delta \rho^{\prime}\right)^{2} .
\end{aligned}
$$

Therefore the functional measure becomes

$$
\mathcal{D}_{g} \rho \cong d \rho_{0} \mathcal{D}_{\bar{g}} \rho^{\prime}\left(\int d^{2} \xi \sqrt{\bar{g}}\right)^{\frac{1}{2}}=d \rho_{0} \mathcal{D}_{\bar{g}} \rho^{\prime}\left(\frac{2 A}{\gamma}\right)^{\frac{1}{2}}
$$

A similar formula holds for the measure of $\phi$. The $\rho_{0}$ integral can be evaluated as

$$
\int d \rho_{0} \delta\left(\int d^{2} \xi \sqrt{\hat{g}} \mathrm{e}^{2(\rho-\phi)}-A\right) \mathrm{e}^{2 \gamma \chi\left(\rho_{0}-\phi_{0}\right)^{2}} \cong \frac{1}{2 A}
$$

while the $\phi_{0}$ integral gives an $A$-independent constant. The integrals of the nonzero modes $\rho^{\prime}, \phi^{\prime}$ give functional determinants of differential operators

$$
\operatorname{det}^{\prime}\left(\Delta_{\bar{g}}-\frac{2 \pi \gamma \chi}{A}\right)^{-\frac{1}{2}} \operatorname{det}^{\prime}\left(\Delta_{\bar{g}}+\frac{2 \pi \gamma \chi}{A}\right)^{-\frac{1}{2}}
$$


where the primes denote an exclusion of the zero mode of $\Delta_{\bar{g}}$. They are the same type of operators as those appearing in the semiclassical analysis of the ordinary Liouville theory [8, 9]. The $A$-dependence is given by [8, 9]

$$
A^{\frac{1}{2}} \operatorname{det}^{\prime}\left(\Delta_{\bar{g}}+\frac{4 \pi \eta \chi}{A}\right)^{-\frac{1}{2}}=\text { const. } \times A^{\frac{1}{12} \chi+\frac{1}{2} \eta \chi} \mathrm{e}^{-K A}
$$

where $K$ is a regularization dependent constant, which we choose to be zero.

Collecting the above results we finally obtain the $A$-dependence of the area-fixed partition function

$$
\tilde{Z}_{\chi}(A)=\text { const. } \times A^{\Gamma(\chi)-3} \mathrm{e}^{-\frac{\mu}{\pi} A}
$$

where the string susceptibility is given by

$$
\Gamma(\chi)=\frac{c-24}{12} \chi+2+O\left((-c)^{-1}\right)
$$

The $\mu$-dependence of the partition function (2) is obtained from eq. (23) by integrating over $A$

$$
Z_{\chi}(\mu)=\text { const. } \times \mu^{-\Gamma(\chi)+2}
$$

Let us compare this result with that of ref. [4]. It was argued in ref. [4] that the functional integral (2) can be reduced to

$$
Z_{\chi}(\mu)=\int \frac{[d \tau]}{V_{\mathrm{CKV}}} \int \mathcal{D}_{\hat{g}} \chi \mathcal{D}_{\hat{g}} \Omega \mathrm{e}^{-S_{\mathrm{CFT}}}
$$

where the action is given by

$$
S_{\mathrm{CFT}}=\frac{1}{8 \pi} \int d^{2} \xi \sqrt{\hat{g}}\left(\hat{g}^{\alpha \beta} \partial_{\alpha} \chi \partial_{\beta} \chi+2 \sqrt{\kappa} R_{\hat{g}} \chi-\hat{g}^{\alpha \beta} \partial_{\alpha} \Omega \partial_{\beta} \Omega+2 \mu \kappa \mathrm{e}^{\frac{1}{\sqrt{\kappa}}(\chi-\Omega)-1}\right) .
$$

The parameter $\kappa$ is determined by the conformal invariance as $\kappa=\frac{24-c}{12}$. To make the integral of $\Omega$ convergent one has to integrate it along the imaginary direction $\Omega=i \bar{\Omega}$ 
$(\bar{\Omega} \in \mathbf{R})$. The $\mu$-dependence of the partition function can be found by shifting the field as $\chi \rightarrow \chi-\sqrt{\kappa} \ln \mu$ in eq. (26). Thus we find the string susceptibility in this approach

$$
\Gamma_{\mathrm{CFT}}(\chi)=\frac{c-24}{12} \chi+2
$$

which is consistent with our semiclassical result (24).

Finally let us discuss other choices of the functional measures in the path integral. In ref. [3] the measures of the metric and the dilaton were defined using the modified metric $\mathrm{e}^{-2 \phi} g_{\alpha \beta}$, while that of the matter field was defined by $g_{\alpha \beta}$. More generally, one may consider also a modification of the matter measure. Here, we consider the following general situation. Suppose that among the matter-ghost system contributing to the Weyl anomaly proportional to $\gamma$, a fraction $\frac{\gamma_{i}}{\gamma}\left(i=1,2,3, \cdots, \sum_{i} \gamma_{i}=\gamma\right)$ has a measure defined by a modified metric $\mathrm{e}^{2 \alpha_{i} \phi} g_{\alpha \beta}=\mathrm{e}^{2\left(\rho+\alpha_{i} \phi\right)} \hat{g}_{\alpha \beta}$. The Liouville action (8) is then replaced by

$$
\begin{aligned}
S_{\mathrm{L}}^{\prime}= & \sum_{i} \frac{\gamma_{i}}{\gamma} S_{\mathrm{L}}\left[\hat{g}_{\alpha \beta}, \rho+\alpha_{i} \phi\right] \\
= & \frac{1}{2 \pi} \int d^{2} \xi \sqrt{\hat{g}}\left(\gamma \hat{g}^{\alpha \beta} \partial_{\alpha} \rho \partial_{\beta} \rho+\gamma R_{\hat{g}} \rho+a \hat{g}^{\alpha \beta} \partial_{\alpha} \phi \partial_{\beta} \phi\right. \\
& \left.+2 b \hat{g}^{\alpha \beta} \partial_{\alpha} \phi \partial_{\beta} \rho+b R_{\hat{g}} \phi\right),
\end{aligned}
$$

where $a=\sum_{i} \gamma_{i} \alpha_{i}^{2}, b=\sum_{i} \gamma_{i} \alpha_{i}$. The case we have considered so far corresponds to $a=b=0$, while the choice in ref. [3] corresponds to $a=-b=\frac{13}{6}$. The measures of $\rho$ and $\phi$ (9) may also be modified. However, it does not change the result of the string susceptibility in the one-loop approximation.

Using eq. (29) instead of eq. (8) we can repeat the above semiclassical analysis. The saddle point is found to be

$$
\phi_{\mathrm{cl}}=-\frac{1}{2} \ln \frac{\gamma+b}{2}, \quad R_{\bar{g}}=\frac{2 \pi(\gamma+b) \chi}{A}
$$

where $\bar{g}_{\alpha \beta}$ is defined in eq. (12). Integrating the fluctuations around the configuration (30) we find that the area-fixed partition function is given by the same expression 
as before except that the functional determinants (21) are replaced by

$$
\operatorname{det}^{\prime}\left(\Delta_{\bar{g}}-\frac{2 \pi(\gamma+b)^{2} \chi}{(\gamma+a+2 b) A}\right)^{-\frac{1}{2}} \operatorname{det}^{\prime}\left(\Delta_{\bar{g}}+\frac{2 \pi(\gamma+b) \chi}{A}\right)^{-\frac{1}{2}}
$$

Thus we find the string susceptibility

$$
\Gamma(\chi)=\left[\frac{c-24}{12}+\frac{(a+b)(26-c+12 b)}{4(26-c+12 a+24 b)}\right] \chi+2+O\left((-c)^{-1}\right)
$$

The choice in ref. [3] $a=-b=\frac{13}{6}$ gives the same susceptibility as eq. (24), which is consistent with the result of refs. [3, 4]. It is interesting to study the model with other choices of $a$ and $b$ using the method of refs. [3], 4] and compare its result with eq. (32).

The author would like to thank S. Yamaguchi for a collaboration in an early stage of this work. He would also like to thank N. Sakai, Y. Matsumura and T. Uchino for useful discussions on the dilaton gravity. 


\section{References}

[1] C.G. Callan, S.B. Giddings, J.A. Harvey and A. Strominger, Phys. Rev. D45 (1991) R1005.

[2] H. Verlinde, in String Theory and Quantum Gravity '91, eds. J. Harvey et al., (World Scientific, Singapore, 1992).

[3] A. Strominger, Santa Barbara preprint UCSBTH-92-18 (May, 1992).

[4] S.P. de Alwis, Colorado preprint COLO-HEP-280 (May, 1992); A. Bilal and C. Callan, Princeton preprint PUPT-1320 (May, 1992).

[5] J.G. Russo and A.A. Tseytlin, Stanford and DAMTP preprint SU-ITP-92-2, DAMTP-1-1992 (January, 1992); K. Hamada, Tokyo preprint UT-Komaba 92-7 (June, 1992); A. Miković, Queen Mary preprint QMW/PH/92/12 (June, $1992)$.

[6] F. David, Mod. Phys. Lett. A3 (1988) 1651; J. Distler and H. Kawai, Nucl. Phys. B321 (1989) 509.

[7] V.A. Kazakov and A.A. Migdal, Nucl. Phys. B311 (1988/89) 171.

[8] A.B. Zamolodchikov, Phys. Lett. B117 (1982) 87.

[9] S. Chaudhuri, H. Kawai and S.-H.H. Tye, Phys. Rev. D36 (1987) 1148.

[10] V.G. Knizhnik, A.M. Polyakov and A.B. Zamolodchikov, Mod. Phys. Lett. A3 (1988) 819 .

[11] N.E. Mavromatos and J.L. Miramontes, Mod. Phys. Lett. A4 (1989) 1847; E. D'Hoker and P.S. Kurzepa, Mod. Phys. Lett. A5 (1990) 1411; E. D'Hoker, Mod. Phys. Lett. A6 (1991) 745.

[12] A.M. Polyakov, Phys. Lett. B103 (1981) 207; Gauge Fields and Strings, (Harwood Academic Publishers, Chur, 1987). 